\title{
Breast augmentation: reducing postoperative nausea and vomiting. a prospective study
}

\begin{abstract}
Background: Breast augmentation is the plastic surgery procedure associated with the greatest risk of postoperative nausea and vomiting (PONV). The purpose of this study is to evaluate the efficacy of a specific antiemetic protocol applied in breast augmentation in order to diminish the incidence of PONV

Methods: A prospective-level of evidence II-study was performed in 247 patients that underwent breast augmentation from October 2010 to October 2014. General anesthesia was induced with propofol, fentanyl and rocuronium. Anesthetic maintenance was accomplished with total intravenous anesthesia and air with oxygen at $50 \%$. All patients received metoclopramide $10 \mathrm{mg}$ intravenously (iv) and $8 \mathrm{mg}$ of ondasetron iv (2mg at the induction and $6 \mathrm{mg}$ over 30 minutes). Intergroup comparison of means was performed using unpaired $t$ tests and analysis of variance, where applicable.

Results: Nausea and emesis occurred with an overall frequency of $4.9 \%$ and $2.8 \%$ respectively. The 95.1 percent of the patient didn't complaint having nausea on the day of operation, and only $3(1.2 \%)$ of them had this symptom remaining until the fifth post op day. Only 7 patients reported vomiting on the day of the operation. There is no significant difference in nausea between smokers and no smokers $(p=0.510)$.

Conclusion: The specific antiemetic protocol applied in breast augmentation operations seems to be highly effective. The fact that we observed no difference between smokers and nonsmokers regarding the incidence of PONV should be further investigated since till now a protective role for smoking against PONV was suggested.
\end{abstract}

Volume 7 Issue 6 - 2017

\author{
Keramidas E,' Vasileiou I, ${ }^{2}$ Pascovitis A, ${ }^{3}$ \\ Liakopoulos D, ${ }^{4}$ Rodopoulou $S^{5}$ \\ 'Plastic Surgeon, Kosmesis Aesthetic Plastic Surgery Center, \\ Greece \\ 2PhD Anesthesiologist Central Clinic of Athens, Greece \\ ${ }_{3}^{3}$ MD Anesthesiologist Central Clinic of Athens, Greece \\ ${ }^{4}$ Vascular Surgeon, Mater Misericordiae University Hospital, \\ Dublin \\ ${ }^{5}$ Plastic Surgeon, EBOBRAS, Kosmesis Aesthetic Plastic Surgery \\ Center,Athens, Greece
}

\begin{abstract}
Correspondence: Vasileiou loanna, PhD Anesthesiologist Central Clinic of Athens, Tsakalof I, Agia Paraskevi,Athens, Greece, Email tzovasileiou@hotmail.com
\end{abstract}

Received: April 28, 2017| Published: May 08, 2017

\section{Introduction}

Postoperative nausea and vomiting (PONV) are common adverse effects that occur after several types of surgical procedures. The general incidence of vomiting is about $30 \%$, the incidence of nausea is about $50 \%$, and in high-risk patients, the PONV rate can be as high as $80 \% \cdot{ }^{1-3}$ The mechanism of PONV is multifactorial and not well-deciphered. The emesis reflex is coordinated by the vomiting center, located in the hindbrain within the medulla oblongata in close proximity to the chemoreceptor trigger zone within the area postrema. Stimuli from peripheral organs and tissues, including the genitalia are transmitted to the vomiting center by the afferent neurons of the vagus nerve. $^{4}$

However, not all patients have the same risk profile for PONV. The incidence of PONV varies with patient-related factors, the nature of surgery performed, type of anesthesia used, and duration of surgery and perioperative management of the patient (Table 1). ${ }^{5}$ The risk of PONV in adult women has been found to be two to three times greater than in adult men. Progesterone or serum gonadotropin seem to be the contributing factor in the higher incidence of PONV in women. ${ }^{5,6}$ Furthermore, obese patients, non-smokers and patients with high levels of anxiety experience more emesis. ${ }^{7}$ Plastic surgery procedures and especially breast augmentation are associated with the greatest risk for PONV. ${ }^{2}$ Over $40 \%$ of these patients develop PONV in the immediate postoperative period or within $24 \mathrm{~h}$ after surgery. ${ }^{2}$

In summary, the purpose of this study is to evaluate the efficacy of a specific antiemetic protocol that our plastic surgery team applied in breast augmentation operations, as well as to determine the possible risk factors for PONV that might be specific to plastic surgery patients, in order to organize an effective prophylactic regimen to avoid these complications.

\section{Materials and methods}

A prospective study was performed in 247 patients that underwent bilateral breast augmentation from October 2011 to October 2014. All patients had the implant behind the pectoralis major muscle (Dual plane technique). Patients signed an informed consent for participation to this study. For the purposes of this study PONV was defined as documented emesis or retching. It was also investigated the post operative pain and the time to perform the operation. It was asked from the patients to describe their pain-symptoms in a ten based scale, prescribing as zero when there is no pain and ten as severe pain. Demographic data were obtained through a preoperative questionnaire and chart review.

The anesthesiologists of our plastic surgery service applied a specific protocol for induction as well as for the prevention of PONV. Using standard monitoring general anesthesia was induced with propofol $(2 \mathrm{mg} / \mathrm{kg})$, fentanyl $(2 \mathrm{mcg} / \mathrm{kg})$ and rocuronium $(0.7 \mathrm{mg} / \mathrm{kg})$ .Anesthetic maintenance was accomplished with total intravenous anesthesia (propofol $4-10 \mathrm{mg} / \mathrm{kg} / \mathrm{h}$, remifentanil $0.25 \mathrm{mcg} / \mathrm{kg} / \mathrm{min}$ ) and air with oxygen at $50 \%$. All patients received metoclopramide $10 \mathrm{mg}$ intravenously (iv) at the induction and $8 \mathrm{mg}$ of ondasetron iv (2mg at the induction and $6 \mathrm{mg}$ were administrated slowly over 30 minutes). High risk patients received also dexamethasone $8 \mathrm{mg}$ shortly after the induction. As high risk patients were stratified obese young ( $>22$ years old) non-smoker women with previous history of nausea and vomiting.

Post operatively, non-steroidal anti-inflammatory drugs and paracetamol was prescribed in all patients. Ondansetron $4 \mathrm{mg}$ iv was delivered 4-6hours after the operation. Patients started drinking water and were mobilized 4 hours after the operation. The PONV on the day of the surgery and the following four days as well as the duration of the procedure as an independent parameter was evaluated. 
All data for descriptive statistical analysis are reported as mean \pm standard deviation (SD). P-values of less than 0,05 were considered as statistically significant. In addition, a comparison was made with proportions compared using Chi-square tests. The mean values of the subgroups were compared using t-test or Mann U Whitney tests as appropriate. To assess relationship between the variables Spearman's rank correlation coefficient was used. All results were analyzed using IBM SPSS (V 21, Statistical Package for the Social Sciences, Inc., Chicago IL).

\section{Results}

The average patient age was $31.7( \pm 16,67)$ years old and weight $55.13 \mathrm{Kg}( \pm 12,86)$. The average duration was 45.94 minutes (minimum 30min -maximum 90min) and the average implant size was 348,18 (225-550). Nausea occurred with an overall frequency of $4.9 \%$ (12 patients) on the day of the surgery and on the second postoperative day 6 patients $(2.4 \%)$ still had nausea. Only 4 patients $(1.6 \%)$ felt nausea on the third as well as on the fourth postoperative day, while 3 patients (1.2\%) still had nausea five days after the surgery (Table 1). Regarding vomiting, 7 patients $(2.8 \%)$ presented with vomiting on the day of the surgery and 2 patients $(0.8 \%)$ on the second postoperative day. On the third day, only one patient $(0.4 \%)$ had vomiting due to the operation while no patient referred vomiting on the fifth postoperative day (Table 2). Regarding nausea and smoke (Table 3), it seems that smoking is not correlated with nausea on any postoperative day ( $\mathrm{p}$-value 0,978$)$. Moreover, there was no correlation regarding smoking and vomiting (Table 4$)\left(\mathrm{X}^{2}\right.$ p-value 0,985$)$.

Table I Post- operative nausea

\begin{tabular}{llllll}
\hline & Day I & Day 2 & Day 3 & Day 4 & Day 5 \\
\hline $\mathrm{n}$ & $\mathrm{I} 2$ & 6 & 4 & 4 & 3 \\
$\mathrm{P}$-value & 0.026 & 0.405 & 0.703 & 0.734 & 0.725 \\
\hline
\end{tabular}

Table 2 Post- operative Vomiting

\begin{tabular}{|c|c|c|c|c|c|}
\hline & Vomiting Day I’ & Vomiting Day 2 & Vomiting Day 3 & Vomiting Day 4 & Vomiting Day 5 \\
\hline $\mathrm{n}$ & 7 & 2 & 1 & 1 & 0 \\
\hline $\begin{array}{l}\text { There are seven groups of antiemetic } \\
\text { agents including phenothiazines, } \\
\text { butyrophenones, benzamides, } \\
\text { corticosteroids, anticholinergics, } \\
\text { antihistamines and 5-HT3 antagonists. } \\
\text { Despite great advances in modern } \\
\text { medicine, there is no single agent } \\
\text { that is completely effective against all } \\
\text { cases of PONV.This is unfortunate } \\
\text { because the sequelae of emesis } \\
\text { and retching can have serious } \\
\text { consequences especially for plastic } \\
\text { surgery operations apart from the } \\
\text { sense of discomfort. Aspiration, } \\
\text { hematoma and wound dehiscence are } \\
\text { some of the potential problems that } \\
\text { might raise the necessity for return } \\
\text { in the operatory room and certainly } \\
\text { lower the patients' satisfactory rate } \\
\text { (Table 2). p-value }\end{array}$ & 0.887 & 0.141 & 0.715 & 0.922 & 0.925 \\
\hline
\end{tabular}

Table 3 Smoking and post-operative nausea

\begin{tabular}{llllll}
\hline & Nausea Day I & Nausea Day 2 & Nausea Day 3 & Nausea Day 4 & Nausea Day 5 \\
\hline Smoker & 4 & 3 & 3 & 3 & 2 \\
Non smoker & 8 & 3 & $\mathrm{I}$ & $\mathrm{I}$ & $\mathrm{I}$ \\
P-value & 0.565 & 0.701 & 0.315 & 0.314 & 0.576 \\
\hline
\end{tabular}

Statistical analysis $\mathrm{X}^{2}, \mathrm{p}<0.05$

Table 4 Smoking and post-operative Vomiting

\begin{tabular}{llllll}
\hline & Vomiting Day I & Vomiting Day 2 & Vomiting Day 3 & Vomiting Day 4 & Vomiting Day 5 \\
\hline Smoker & 3 & $\mathrm{I}$ & $\mathrm{I}$ & $\mathrm{I}$ & $\mathrm{I}$ \\
Non smoker & 4 & $\mathrm{I}$ & 0 & 0 & 0 \\
p-value & 0.985 & 0.83 & $0.35 \mathrm{I}$ & 0.244 & 0.276 \\
\hline
\end{tabular}

Statistical analysis $\mathrm{X}^{2}, \mathrm{p}<0.05$

57 patients $(23.1 \%)$ felt moderate to severe pain on the day of the surgery, since they categorized their pain over 5 in the 10 scale. 190 patients $(76.9 \%)$ reported pain score from 5 to 1 in a scale of 10 , while 14 patients $(5.7 \%)$ didn't feel any pain on the day of the surgery. The complaints regarding pain were steadily reduced. On the second postoperative day 56 patients complained about moderate and severe pain. On the third and fourth postoperative day 207 (83.8\%) and 210
$(89.9 \%)$ patients respectively, reported a pain score from 0 to 5 in a scale of 10 . On the fifth postoperative day 96 patients $(38,9 \%)$ did not feel any pain and only 25 patients $(10,1 \%)$ had moderate to severe complaints about pain. Regarding analgesia the vast majority of the women $(93.5 \%)$, received paracetamol, NSAIDs or combination of them to alleviate the pain and $4.0 \%$ didn't receive any kind of pain killers. We performed Mann Whitney U-test and we didn't find any 
significant difference regarding the pain sensation between smokers and non-smokers (p-value 0.268). The smoking habits don't seem to affect the consumption of analgesics ( $p$ value 0,299 ).

No significant correlation $(r h o=0.014, p=0,832$ ) was found between operation time and pain as well as for pain on each post op day and implant size $(p>0,05)$. We investigated the relation between pain and PONV. We divided the patients into two groups. In group one were patients with pain score from 5 to zero in the scale of 10 . In group two were patients with pain score from 5 to 10 . Then we performed t-test between the patient in group one and group two regarding pain and PONV. No statistically significant correlation was found between PONV and pain score less than 5 . We concluded that there is statistically significant difference on the first postoperative day between nausea and a pain score over five on the same day $(\mathrm{p}=0.006)$. No statistically difference was mentioned for the other postoperative days between pain and PONV.

We performed correlation Spearman's rho test and we found that there is a correlation between nausea on the first day and the size of the implant $(\mathrm{p}=0,015)$, but there is no correlation of nausea and implant on the second $(\mathrm{p}=0.268)$, third $(\mathrm{p}=0,818)$, fourth $(\mathrm{p}=0.818)$ and fifth post op day $(\mathrm{p}=0,994)$.

\section{Discussion}

The overall incidence of PONV in plastic surgery is estimated around 35 percent. $^{8}$ The plastic surgery procedure associated with the greatest risk of PONV is breast augmentation. The incidence of PONV for breast augmentation is eight to ten times higher than for other types of plastic surgery 2]. Four statistically significant factors have been identified that place plastic surgery patients at increased risk for developing PONV; female gender, postoperative opioid use, use of general anesthesia and duration of surgery 89] . Regarding the duration of surgery, it has been reported that for every 30-minutes increase in the duration of surgery, the risk for PONV increases by $60 \% .^{2}$ In our study the mean surgical time was 45.9 minutes, which means that its effect on the incidence of PONV was minimal. Plastic surgery patients' satisfaction is based on overall experience as much as the final result, consequently the prevention of PONV is of great importance.

Gan et al., ${ }^{10}$ reported in 2003 guidelines for the management of $\mathrm{PONV}^{10}$ and the first step according to these guidelines is to identify patients at high risk for PONV. Once this risk is established then the next steps are to reduce the risk factors and prescribe antiemetic prophylaxis. Ondasetron is a mainstay in the armamentarium for "fighting" nausea. It is a multi-examined agent regarding its usefulness as prophylactic antiemetic but it is not the only one with desirable efficacy. However, several studies have reported improved efficacy of ondasetron over metoclopramide and droperidol. ${ }^{11-13}$ Ondasetron is a 5HT3 receptor antagonist that block the receptors in the chemotactic trigger zone (CTZ) as well as vagal nerve terminals and has a negligible side-effects profile. ${ }^{14}$ Marcus et al., ${ }^{9}$ reported that ondasetron reduced the incidence of postoperative emesis and the perception of nausea severity. ${ }^{9}$ Metoclopramide, a gastrointestinal motility stimulant that blocks dopaminergic receptors both peripherally and centrally in the $\mathrm{CTZ}$, increases the lower esophageal sphincter tone and aids in gastric emptying. Several studies however, have suggested improved efficacy of ondasetron over metoclopramide. ${ }^{12,3,15}$ Dexamethasone on the other hand belongs to corticosteroids. The antiemetic effect is due to their anti-inflammatory or membrane-stabilizing effects, as well as due to the inhibition of prostaglandin synthesis and control of endorphin release. ${ }^{6,16}$
Combination of drugs from different categories has been found to be more effective due to the fact that it blocks the vomiting reflex at different sites. ${ }^{17,18}$ The findings of our study are in accordance with this since we reported that a multiple drug regimen showed great efficacy in the prolepsis of PONV. 235 patients (95.1\%) did not suffer from nausea the day of the surgery, while only 7 patients $(2.8 \%)$ suffered from vomiting. Steely et al in a case series of 143 plastic surgery patients reported that the incidence of PONV was reduced to $3 \%$ with a multiple drug regimen. The antiemetic protocol included ondasetron $4 \mathrm{mg}$ followed by metoclopramide $10 \mathrm{mg}$ iv and promethazine $25 \mathrm{mg}$ intramuscularly 8]. Moreover, dexamethasone has been reported to be particularly effective when used in combination with 5-HT3 receptor antagonists as it may i)reduce the levels of serotonin by depleting its precursor tryptophan, ii)prevent release of serotonin in the gut and iii) sensitizes the 5-HT3 receptors to other antiemetics. ${ }^{16}$ Karanicolas et al. ${ }^{19}$ in a meta-analysis of 17 trials found that dexamethasone reduced the incidence of postoperative nausea by $41 \%$, vomiting $59 \%$ and nausea or vomiting $45 \% .{ }^{19}$ Regarding metoclopramide, although there is a lack of evidence for antiemetic efficacy, it has been found that it is useful in preventing the delayed gastric emptying caused by opioids due to its ability to promote gastric motility. ${ }^{20}$ In our study the concept was to try to minimize immediate as well as delayed PONV and to improve patients' satisfaction. As our results indicate our targets were achieved.

In addition, propofol total intravenous anesthesia (TIVA) may have also contributed to the reduction in PONV incidence in our study. It has been found that patients who receive propofol at the induction tend to have less PONV. ${ }^{21}$ Moreover, it has been reported that propofol TIVA resulted in a significant reduction of PONV as compared with inhaled anesthesia. ${ }^{22}$ The inhibitory effect of propofol on 5-HT3 receptors may be responsible for these anti-emetic properties of propofol 23].

Pain itself may have been a confounding factor in the genesis of the detected PONV. It is known that pain is associated with PONV and that opioids, rather than acting as a causative factor, may help to ameliorate its severity. ${ }^{24}$ Palazzo and Evans however, recognized postoperative opioid use as an important factor associated with PONV 25]. In our study there was no need for postoperative opioid analgesic; thus we limited the influence of this predisposing factor. The first day $5.7 \%$ of the patients did not feel any pain, while $76.9 \%$ reported a pain score from 1 to 5 out of 10 . The specific surgical technique definitely has contributed to these results.

Patient satisfaction is very important in plastic surgery, especially in cases of elective procedures like breast augmentation. PONV has been listed as the most undesirable scenario postoperatively, followed by pain and sedation. ${ }^{26} \mathrm{PONV}$ is also a limiting factor in the timely discharge of ambulatory patients with significant economic consequences. Tramer et al., ${ }^{27}$ suggested that use of ondasetron as a prophylactic measure is not a cost-effective measure for controlling emesis when compared with rescue therapy 27]. This may be true; however we suggest that the drug costs associated with the prevention of PONV are minor relative to the overall expense of managing patients once they develop POVV. Taking into consideration the fact that only $3 \%$ of the total cost of managing a patient with PONV is spent on antiemetic treatment, the use of prophylactic antiemetic medications can be a cost-effective measure in preventing PONV. ${ }^{18}$ In our study none of our patients needed to extend hospital stay due to PONV.

Interestingly, our study contradicted the fact that smoking has an anti-emetic effect. It has been reported that cigarette confers protection against PONV due to presence of an anti-emetic substance 
in tobacco smoke. ${ }^{28}$ A number of epidemiological studies have shown that women who smoke during pregnancy are affected by nausea of pregnancy to a much lesser degree than those who do not 29]. The pharmacological receptors which mediate PONV are known to act at the dopamine (D2), cholinergic, histamine (H1), 5-HT3 and NK1 receptors and if there were an antemetic in cigarette smoke it would have to belong to one of these classes of receptor-blocking drugs. Alternatively, the reduction in PONV may be seen as an adaptive response to a repeated emetic stimulus, although it is clear that usually only the uninitiated or those who have consumed excessive amounts find tobacco emetogenic. Our study however showed that there was no difference regarding the incidence of postoperative nausea between smokers and nonsmokers $(\mathrm{p}=0.594)$; there was no statistically important difference between two groups even the first postoperative day $(p=0.365)$. Smokers and no-smokers did not show any difference regarding the presence of vomiting $(\mathrm{p}=0.465)$ Detoxifcation of most drugs occurs via the P450 (CYP) enzyme pathways. In humans, the CYP isoenzymes, which are responsible for drug metabolism belong to families 1-4. Cigarette smoking can result in as much as a 3-fold increase in CYP1A2 activity from induction. CYP1A2 is the major enzyme involved in the metabolism of a number of drugs, some of which are commonly used by anesthetists. ${ }^{30}$ In contrast, volatile anesthetics are metabolized by CYP2E1 which is also induced by nicotine 31]. A patient whose liver enzymes, in particular CYP2E1, have been induced as a result of smoking would be expected to have a correspondingly higher rate of metabolism of the respective volatile agent. Such a patient might have a quicker and smoother recovery without PONV. Enzyme induction is an important mechanism that may influence the breakdown of a number of volatile and non-volatile anesthetic agents. The extent to which liver enzymes are induced will determine the rate and possibly the quality of recovery. There are interindividual, racial, and sex-related differences in the activity of liver enzymes including CYP2E1, and this could explain some of the differences in recovery from anesthesia. There are a number of pharmacological, dietary, and environmental substances including cigarette smoke that can induce or inhibit CYP1A2 and CYP2E1. The finding of our study, that there is no difference in the incidence of PONV between smokers and nonsmokers, suggests that some of the pharmacological factors that we used inhibit the effects of smoking on CYP2E1 and CYP1A2.

\section{Conclusion}

In conclusion, the results of this study indicate that the specific antiemetic protocol that was applied to all patients undergoing breast augmentation combined with the specific surgical technique ${ }^{32}$ and the minor surgical time, was highly effective. Our purpose to minimize immediate as well as delayed PONV and to improve patients' satisfaction was achieved. The fact that we observed no difference between smokers and nonsmokers regarding the incidence of PONV should be further investigated since till now all studies suggest a protective role for smoking against PONV.

\section{Acknowledgements}

None.

\section{Conflicts of interest}

Author declares there are no conflicts of interest.

\section{Funding}

None.

\section{References}

1. Apfel CC. A simplified risk score for predicting postoperative nausea and vomiting: conclusions from cross-validations between two centers. Anesthesiology, 1999:91(3):693-700.

2. Sinclair DR, FChung, G Mezei. Can postoperative nausea and vomiting be predicted? Anesthesiology, 1999:91(1):109-118.

3. FortierJF. Chung, and J. Su, Unanticipated admission after ambulatory surgery--a prospective study. Can J Anaesth, 1998;45(7): 612-619.

4. Borison HL. Area postrema: chemoreceptor circumventricular organ of the medulla oblongata. Prog Neurobiol, 1989;32(5):351-390.

5. Watcha MF,P F.White, Postoperative nausea and vomiting. Its etiology, treatment, and prevention. Anesthesiology. 1992;77(1):162-184.

6. LeTP,TJ.Gan. Update on the management of postoperative nausea and vomiting and postdischarge nausea and vomiting in ambulatory surgery. Anesthesiol Clin. 2010;28(2): 225-249.

7. Yogendran S, et al. A prospective randomized double-blinded study of the effect of intravenous fluid therapy on adverse outcomes on outpatient surgery. Anesth Analg, 1995;80(4):682-686.

8. Steely RL, et al. Postoperative nausea and vomiting in the plastic surgery patient. Aesthetic Plast Surg. 2004;28(1): 29-32.

9. MarcusJR. et al. The prevention of emesis in plastic surgery: a randomized, prospective study. Plast Reconstr Surg, 2002;109(7):2487-2494.

10. Gan TJ, et al. Consensus guidelines for managing postoperative nausea and vomiting. Anesth Analg, 2003;97(1): 62-71.

11. Domino KB.,et al. Comparative efficacy and safety of ondansetron, droperidol, and metoclopramide for preventing postoperative nausea and vomiting: a meta-analysis. Anesth Analg, 1999;88(6): 1370-1379.

12. Dabbous A.et al.Post-laparoscopic vomiting in females versus males: comparison of prophylactic antiemetic action of ondansetron versus metoclopramide. JSLS. 1998;2(3):273-276.

13. MalinsAF. et al. Nausea and vomiting after gynaecological laparoscopy: comparison of premedication with oral ondansetron, metoclopramide and placebo. Br J Anaesth. 1994;72(2): 231-233.

14. PearmanMH. Single dose intravenous ondansetron in the prevention of postoperative nausea and vomiting. Anaesthesia. 1994;49:11-5.

15. Paxton LD, ACMcKay, RK. Mirakhur. Prevention of nausea and vomiting after day case gynaecological laparoscopy. A comparison of ondansetron, droperidol, metoclopramide and placebo. Anaesthesia. 1995;50(5): 403-406.

16. Henzi I, B. Walder, MR. Tramer, Dexamethasone for the prevention of postoperative nausea and vomiting: a quantitative systematic review. Anesth Analg, 2000;90(1): 186-194.

17. Khalil S, et al. Ondansetron/promethazine combination or promethazine alone reduces nausea and vomiting after middle ear surgery. $J$ Clin Anesth. 1999;11(7):596-600.

18. Sweis I, SS Yegiyants, MN Cohen. The management of postoperative nausea and vomiting: current thoughts and protocols. Aesthetic Plast Surg, 2013;37(3): 625-633.

19. Karanicolas PJ. et al. The impact of prophylactic dexamethasone on nausea and vomiting after laparoscopic cholecystectomy: a systematic review and meta-analysis. Ann Surg, 2008;248(5): 751-762.

20. DeBalli P. The use of propofol as an antiemetic. Int Anesthesiol Clin. 2003;41(4):67-77.

21. Gupta A, et al. Comparison of recovery profile after ambulatory anesthesia with propofol, isoflurane, sevoflurane and desflurane: a systematic review. Anesth Analg, 2004;98(3): 632-641. 
22. Goll,V, et al. Ondansetron is no more effective than supplemental intraoperative oxygen for prevention of postoperative nausea and vomiting. Anesth Analg, 2001;92(1): 112-117.

23. Vasileiou I, et al. Propofol: a review of its non-anaesthetic effects. Eur J Pharmacol. 2009;605(1-3):1-8.

24. Andersen R,K Krohg. Pain as a major cause of postoperative nausea. Can Anaesth Soc J. 1976;23(4):366-369.

25. Palazzo M, R Evans. Logistic regression analysis of fixed patient factors for postoperative sickness: a model for risk assessment. Br J Anaesth. 1993;70(2):135-140.

26. EberhartLH, et al. The development and validation of a risk score to predict the probability of postoperative vomiting in pediatric patients. Anesth Analg. 2004;99(6):1630-1637.

27. Tramer MR.et al. Cost-effectiveness of ondansetron for postoperative nausea and vomiting. Anaesthesia. 1999;54(3):226-234.
28. Hough M,B Sweeney. The influence of smoking on postoperative nausea and vomiting. Anaesthesia. 1998;53(9):932-933.

29. GadsbyR, AM Barnie-Adshead, C. Jagger.Pregnancy nausea related to women's obstetric and personal histories. Gynecol Obstet Invest. 1997;43(2):108-111.

30. Tanaka E. Clinically important pharmacokinetic drug-drug interactions: role of cytochrome P450 enzymes. J Clin Pharm Ther. 1998;23(6): 403-416.

31. ZevinS,NL.Benowitz. Drug interactions with tobacco smoking. An update. Clin Pharmacokinet, 1999;36(6):425-438.

32. KeramidasE. Zero breast implant infection rate and $0.05 \%$ hematoma rate following 1720 silicone implant placements for primary breast augmentation: surgical technique or surgeon luck? Aesthetic Plast Surg, 2009;33(1): 123-124. 